\title{
Nano and micro Hall-effect sensors for room-temperature scanning hall probe microscopy
}

\author{
A. Sandhu ${ }^{a, *}$, A. Okamoto ${ }^{b}$, I. Shibasaki $^{b}$, A. Oral $^{c}$ \\ ${ }^{a}$ Research Centre for Quantum Effect Electronics, Tokyo Institute of Technology, 2-12-l, O-okayama, \\ Meguro-ku, Tokyo, 152-8552, Japan \\ b Asahikasei Corporation, 2-1, Samejima, Fuji City, 416-8501 Japan \\ ${ }^{\mathrm{c}}$ Department of Physics, Bilkent University, 06533 Ankara, Turkey
}

Available online 2 April 2004

\begin{abstract}
GaAs/AlGaAs two-dimensional electron gas (GaAs-2DEG) Hall probes are impractical for sub-micron roomtemperature scanning Hall microscopy (RT-SHPM), due to surface depletion effects that limit the Hall driving current and magnetic sensitivity $\left(B_{\min }\right)$. Nano and micro Hall-effect sensors were fabricated using $\mathrm{Bi}$ and InSb thin films and shown to be practical alternatives to GaAs-2DEG probes for high resolution RT-SHPM. The GaAs-2DEG and InSb probes were fabricated using photolithography and the Bi probes by optical and focused ion beam lithography. Surface depletion effects limited the minimum feature size of GaAs-2DEG probes to $\sim 1.5 \mu \mathrm{m}^{2}$ with a maximum drive current $I_{\max }$ of $\sim 3 \mu \mathrm{A}$ and $B_{\min } \sim 0.2 \mathrm{G} / \sqrt{\mathrm{Hz}}$. The $B_{\min }$ of $1.5 \mu \mathrm{m}^{2} \mathrm{InSb}$ Hall probes was $6 \times 10^{-3} \mathrm{G} / \sqrt{\mathrm{Hz}}$ at $I_{\max }$ of $100 \mu \mathrm{A}$. Further, $200 \mathrm{~nm} \times 200 \mathrm{~nm}$ Bi probes yielded good RT-SHPM images of garnet films, with $I_{\max }$ and sensitivity of $40 \mu \mathrm{A}$ and $\sim 0.80 \mathrm{G} / \sqrt{\mathrm{Hz}}$, respectively.
\end{abstract}

(c) 2004 Elsevier B.V. All rights reserved.

Keywords: Hall-effect sensors; Scanning Hall probe microscopy; Magnetic imaging; Ferromagnetic domains

\section{Introduction}

Scanning Hall probe microscopy (SHPM) has been shown to be a valuable tool for the direct and quantitative method for magnetic imaging of localized surface magnetic fluctuations of superconductors and ferromagnetic materials [1-3]. A key element of a SHPM system is the magnetic field

\footnotetext{
${ }^{*}$ Corresponding author. Tel.: +81-35-734-2807; fax: $+81-35$ 734-2807.

E-mail address: sandhu@pe.titech.ac.jp (A. Sandhu).
}

sensor or Hall probe (HP). The magnetic sensitivity of a HP depends on the Hall coefficient of the material and the series resistance of the conducting channels of the 'Hall cross' and its distance from the sample being measured. Thus selection of the material for fabricating the HP is very important in order to obtain high spatial resolution and high magnetic sensitivity. We have previously reported on the use of $\sim 1 \mu \mathrm{m}^{2}$ GaAs/AlGaAs 2DEG heterostructure (GaAs-2DEG) Hall-effect sensors as HPs for room temperature scanning Hall probe microscope (RT-SHPM) [3-7]. In spite of their 
excellent room temperature magnetic sensitivity, the GaAs-2DEG HPs are impractical for high spatial resolution RT-SHPM measurements because their performance is severely degraded for dimensions below $\sim 1.0 \mu \mathrm{m}^{2}$ due to surface depletion effects that limit the maximum drive current $\left(I_{\max }\right)$ and consequently the magnetic sensitivity. Thus alternative materials, not exhibiting such current limiting effects, are required in order to fabricate sub-micron Hall sensors for high spatial resolution magnetic imaging of ferromagnetic domains by room temperature scanning Hall probe microscopy.

In this paper, we describe the results of a comparative study on the performance of nano and micro-Hall sensors fabricated using GaAs2DEG, polycrystalline Bi and single crystal $\mathrm{InSb}$ thin films and show that InSb and Bi thin films are practical alternatives for fabricating high resolution Hall probes for RT-SHPM imaging.

\section{Experimental}

The main components of the RT-SHPM are shown in Fig. 1, including a mini-pulse coil for generating external bias fields of 3T as previously reported [8]. Magnetic imaging is carried out by: (i) lowering the Hall probe into close proximity to the

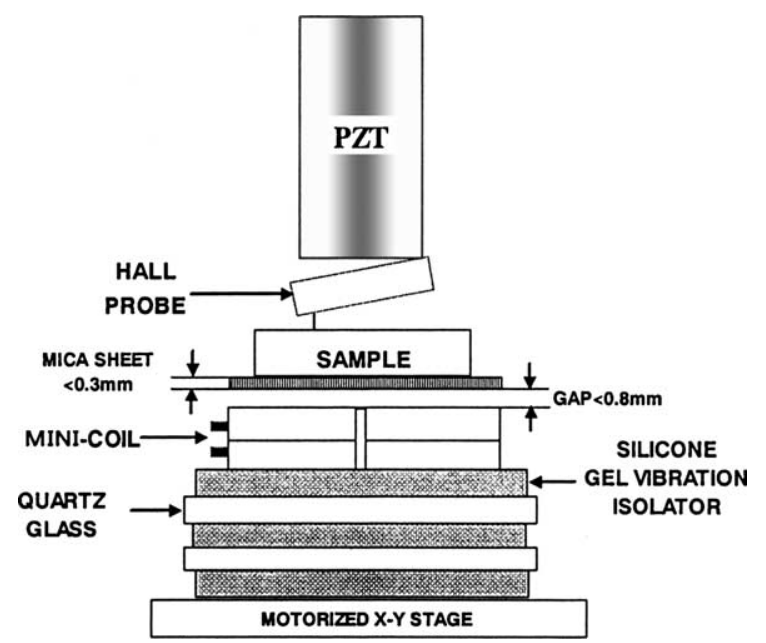

Fig. 1. Main components of room temperature scanning Hall probe microscope with integrated mini-coil for generating pulsed magnetic fields. sample surface until a tunnel current is detected between a STM-tip integrated adjacent to the 'Hallcross' and sample surface; (ii) scanning the Hall probe over the surface whilst monitoring changes in Hall voltage that are proportional to the perpendicular component of the stray fields emanating for the sample surface. In this study, RT-SHPM measurements were carried out with the Hall probes between 0.35 and $0.50 \mu \mathrm{m}$ above the sample surface.

The GaAs-2DEG micro-HPs were fabricated by optical lithography using GaAs/AlGaAs heterostructures grown by MBE with a $300 \mathrm{~K}$ sheet carrier density and mobility of $2 \times 10^{11} \mathrm{~cm}^{2}$ and $4000 \mathrm{~cm}^{2} / \mathrm{Vs}$, respectively. The $2 \mathrm{DEG}$ was located approximately $100 \mathrm{~nm}$ below the epilayer surface.

The InSb micro-HPs were fabricated by optical lithography using $1 \mu \mathrm{m}$ thick epitaxial InSb thin films grown by MBE on a semi-insulating GaAs substrate [9]. The $300 \mathrm{~K}$ carrier concentration and mobility of the InSb epilayers was $2 \times 10^{12} \mathrm{~cm}^{-2}$ and $55,500 \mathrm{~cm}^{2} / \mathrm{Vs}$, respectively. Standard room temperature van der Pauw Hall measurements showed the InSb films to have a Hall coefficient $\left(R_{\mathrm{H}}\right)$ of 0.03 $\Omega / \mathrm{G}$ and a series resistance of $\left(R_{\mathrm{s}}\right)$ of $2.2 \mathrm{k} \Omega$.

The Bi nano-HPs were fabricated using a combination of optical lithography to define $\sim 2.0$ $\mu \mathrm{m} \times 2.0 \mu \mathrm{m}$ 'Hall crosses' followed by focused ion beam milling (FIB) using Ga ions, for defining 200 $\mathrm{nm} \times 200 \mathrm{~nm}$ HP structures. The FIB processing was carried out using a Hitachi FB-2000A system employing an ion current of $15 \mathrm{~A} / \mathrm{cm}^{2}$ and voltage of $30 \mathrm{kV}$.

The final structure of all three types of HPs was similar to the $\sim 1.5 \mu \mathrm{m} \times 1.5 \mu \mathrm{m}$ InSb micro-Hall probe shown in Fig. 2(a), where the 'Hall cross' was located $\sim 10 \mu \mathrm{m}$ from the corner of the chip. Fig. 2(b) shows the $200 \mathrm{~nm} \times 200 \mathrm{~nm}$ Bi Hall probe, produced by FIB milling.

\section{Results and discussion}

The signal to noise ratio (hereafter referred to as 'magnetic sensitivity') of a Hall sensor, can be defined as:

$$
\frac{S}{N}=\frac{\left(I_{\mathrm{H}} R_{\mathrm{H}} B\right)}{\sqrt{4 k_{\mathrm{B}} T R_{\mathrm{S}} \Delta f}},
$$



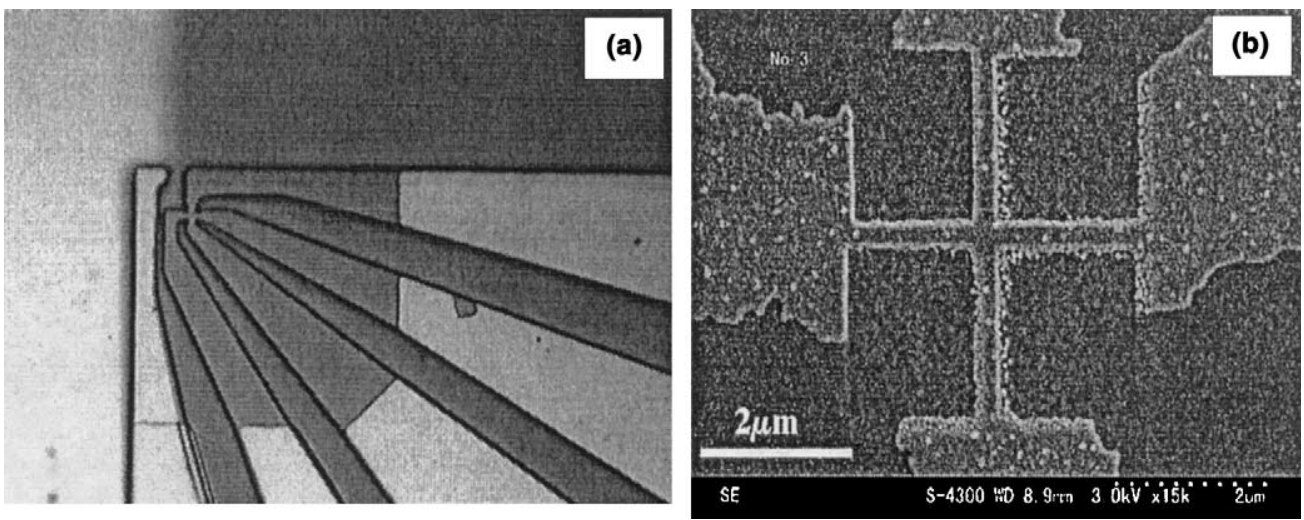

Fig. 2. (a) Optical image of $1.5 \mu \mathrm{m}^{2}$ InSb Hall probe showing STM metallization used for vertical alignment. (b): SEM of 200 $\mathrm{nm} \times 200 \mathrm{~nm}$ Bi Hall probe fabricated by focused ion beam milling.
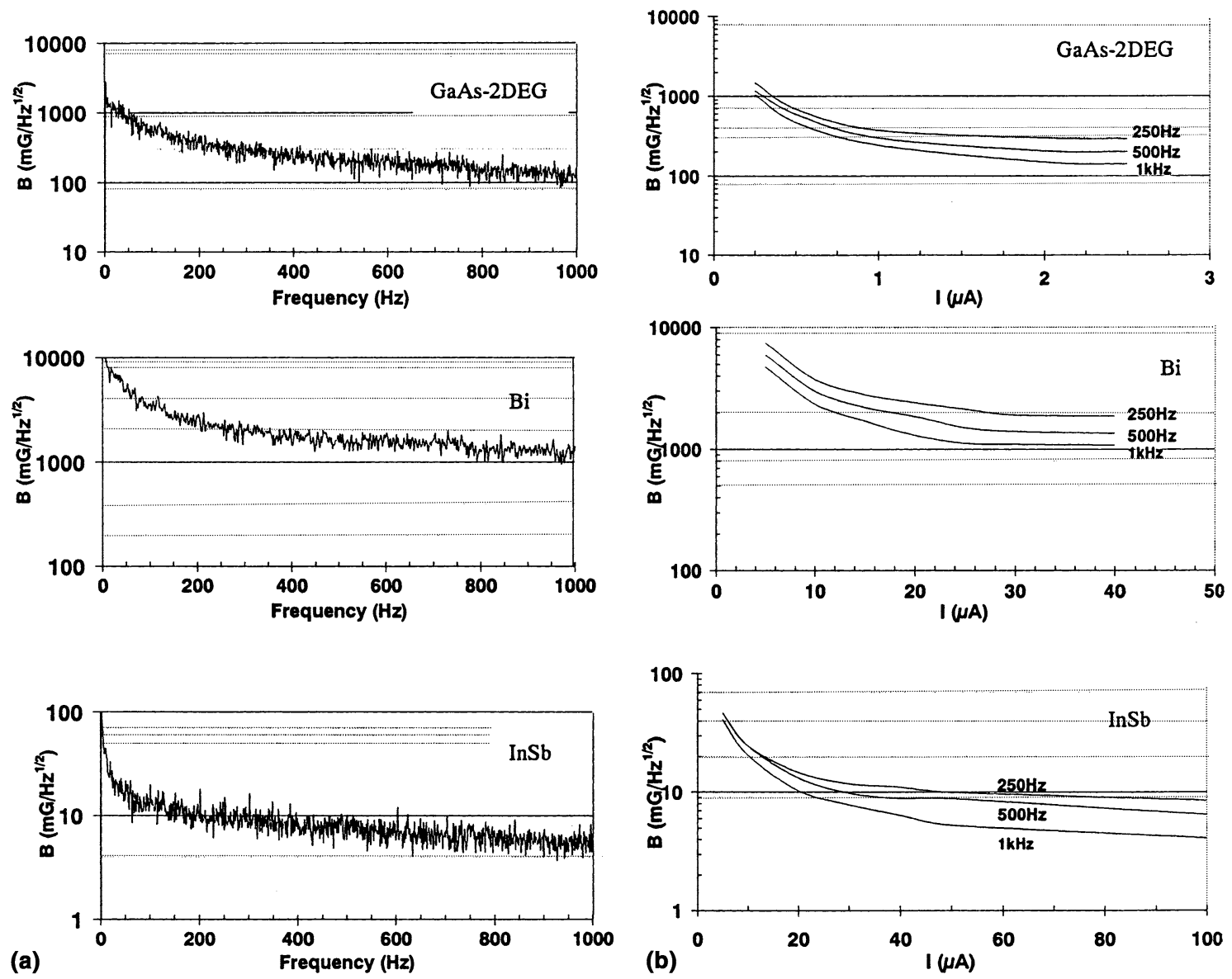

Fig. 3. (a) Frequency and (b) the current dependence of the minimum detectable field for the GaAs-2DEG, Bi, and InSb Hall sensors. 


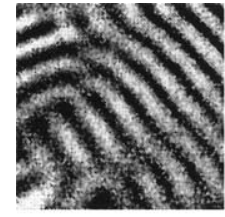

$10 \mu \mathrm{A}$

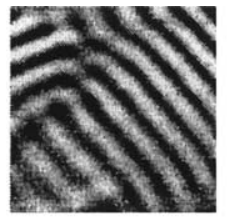

$30 \mu \mathrm{A}$

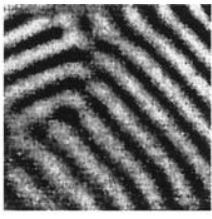

$50 \mu \mathrm{A}$

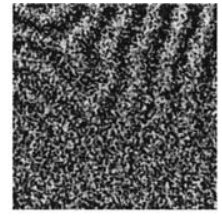

$10 \mu \mathrm{A}$

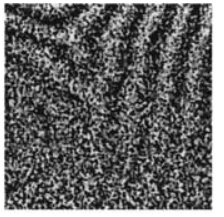

$20 \mu \mathrm{A}$

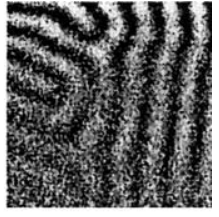

$40 \mu \mathrm{A}$

Fig. 4. Typical $25 \mu \mathrm{m} \times 25 \mu \mathrm{m}$ RT-SHPM images of garnet films for a range of Hall currents measured using: (a) the InSb microsensor; (b) the Bi nano sensor.

where $I_{\mathrm{H}}$ is the Hall driving current, $B$ the magnetic field being measured, $\Delta f$ the measurement band width, $k_{\mathrm{B}}$ Boltzmann's constant, $R_{\mathrm{S}}$ the series resistance, and $T$ the measurement temperature. This relationship between the signal and noise implies that a high sensitivity Hall sensor should have a large current driving capability and a small series resistance. The Hall coefficient of the GaAs2DEG $1.5 \mu \mathrm{m}^{2} \mathrm{HP}$ was the highest of all three fabricated but an $R_{\mathrm{S}}$ of $100 \mathrm{k} \Omega$ and subsequent $I_{\max }$ of only $3 \mu \mathrm{A}$ at room temperature limited the possibility of a further reduction in size without a drastic degradation of the magnetic sensitivity hence limiting the minimum practical HP size to $\sim 1 \mu \mathrm{m}^{2}$ for RT-SHPM imaging.

The noise spectra of the Hall sensors were measured for a range of driving currents using a FFT signal analyzer ( $1 \mathrm{~Hz}$ equivalent bandwidth) with the gain and bandwidth of the RT-SHPM Hall voltage amplifier set at 10,000 and $1 \mathrm{kHz}$, respectively. Fig. 3(a) and (b) show the frequency and current dependence of the minimum detectable field $\left(B_{\min }\right)$ for the GaAs-2DEG, Bi, and InSb Hall sensors. There is a small $1 / f$ noise component in the frequency dependence results. Further, the noise decreases at higher frequencies which we tentatively ascribe as being due to spurious capacitances between the measurement leads and the Hall probe which reduced the bandwidth of the Hall voltage signal. The InSb HP showed the most promising sensitivity with a minimum detectable field of approximately $6-8 \mathrm{mG} / \sqrt{\mathrm{Hz}}$.

Figs. 4(a) and (b) show the drive current dependence of $25 \mu \mathrm{m} \times 25 \mu \mathrm{m}$ RT-SHPM images of a $5.5 \mu \mathrm{m}$ thick crystalline bismuth substituted garnet thin film measured using the $1.5 \mu \mathrm{m} \times 1.5 \mu \mathrm{m} \mathrm{InSb}$ and $200 \mathrm{~nm} \times 200 \mathrm{~nm} \mathrm{Bi} \mathrm{HPs,} \mathrm{respectively.} \mathrm{The}$ black and white regions correspond to surface field variations of $\pm 52 \mathrm{G}$ into and out of the plane of the paper. No differences in the images were observed for Hall currents greater than $40 \mu \mathrm{A}$, using these two types of sensors. These results demonstrate that it is possible to drive the InSb and Bi microHPs with large currents without degradation of HP performance. In particular, in the case of InSb the magnetic sensitivity was improved by a factor of ten compared with the GaAs-2DEG HPs.

\section{Summary}

Bismuth and epitaxial InSb thin films were demonstrated to be practical alternative materials for fabricating sub-micron HPs for high spatial resolution room temperature scanning Hall probe microscopy. Further improvements in the room temperature sensitivity and spatial resolution $(50 \mathrm{~nm})$ can be envisaged by using thinner InSb films and InAs/InSb-2DEG heterostructures.

\section{Acknowledgements}

This work was partly funded by the Ministry of Education, Culture, Sports, Science and Technology of the Japanese Government, Grant in Aid No. 15560271.

\section{References}

[1] A. Oral, S.J. Bending, M. Henini, Appl. Phys. Lett. 69 (1996) 1324-1326.

[2] A. Oral, S.J. Bending, M. Henini, J. Vac. Sci. Technol. B 14 (1996) 1202-1205. 
[3] A. Sandhu, A. Oral, H. Masuda, S.J. Bending, J. Cryst. Growth 227 (2001) 899-905.

[4] A. Sandhu, H. Masuda, A. Oral, S.J. Bending, Jpn. J. Appl. Phys. 40 (2001) L524-L527.

[5] A. Sandhu, H. Masuda, K. Kurosawa, A. Oral, S.J. Bending, Electron. Lett. 37 (2001) 1335-1336.

[6] A. Sandhu, N. Iida, H. Masuda, A. Oral, S.J. Bending, J. Magn. Magn. Mater. 242-245 (2002) 1249-1252.
[7] A. Sandhu, H. Masuda, A. Oral, S.J. Bending, Ultramicroscopy 91 (2002) 97-101.

[8] A. Sandhu, H. Masuda, A. Oral, Jpn. J. Appl. Phys. 41 (2002) L1402-L1405.

[9] I. Shibasaki, A. Okamoto, A. Ashihara, K. Suzuki, in: Technical Digest of the 18th Sensor Symposium, 2001, pp. 233-238. 